PROCEEDINGS OF THE

AMERICAN MATHEMATICAL SOCIETY

Volume 135, Number 12, December 2007, Pages 3989-3994

S 0002-9939(07)08949-6

Article electronically published on September 12, 2007

\title{
A NON-HAUSDORFF MODEL FOR THE COMPLEMENT OF A COMPLEXIFIED HYPERPLANE ARRANGEMENT
}

\author{
NICHOLAS PROUDFOOT \\ (Communicated by Ted Chinburg)
}

\begin{abstract}
Given a hyperplane arrangement $\mathcal{A}$ in a real vector space $V$, we introduce a real algebraic prevariety $\mathcal{Z}(\mathcal{A})$, and exhibit the complement of $\mathcal{A}$ in the complexification of $V$ as the total space of an affine bundle over $\mathcal{Z}(\mathcal{A})$ with fibers modeled on the dual vector space $V^{\vee}$.
\end{abstract}

\section{Statement}

Let $V$ be a real vector space of dimension $d$, and let $f_{1}, \ldots, f_{n}$ be a collection of nonconstant affine linear functions on $V$ such that the associated linear forms span the dual vector space $V^{\vee}$. Let $\mathcal{A}$ denote the collection of affine hyperplanes $H_{1}, \ldots, H_{n} \subseteq V$, where $H_{j}=f_{j}^{-1}(0)$ for all $j \in\{1, \ldots, n\}$. Let

$$
\mathcal{M}(\mathcal{A}):=V^{\mathbb{C}} \backslash \bigcup_{j=1}^{n} H_{j}^{\mathbb{C}}
$$

be the complement of the complexification of $\mathcal{A}$. Our goal is to introduce a new space which is naturally the base of a principal bundle with structure group $V^{\vee}$ and total space $\mathcal{M}(\mathcal{A})$. Note that a principal bundle with structure group equal to a vector space is the same as an affine bundle whose associated vector bundle is trivial.

Our new space, which we call $\mathcal{Z}(\mathcal{A})$, will be a real algebraic prevariety of dimension $d$, where "prevariety" means that $\mathcal{Z}(\mathcal{A})$ will not be Hausdorff in the analytic topology. Though this may sound nasty, our description will be quite simple. We construct $\mathcal{Z}(\mathcal{A})$ by gluing together a collection of vector spaces $\left\{V_{C}\right\}$, each isomorphic to $V$, where the index $C$ ranges over the chambers of $\mathcal{A}$, by which we mean the connected components of the complement of $\mathcal{A}$ in $V$. These vector spaces are attached to each other along open sets, according to the following rule:

$$
V_{C} \cap V_{C^{\prime}} \cong V \backslash \bigcup_{\substack{H_{j} \\ C \text { separates }}} H^{\prime} .
$$

In Figure 1 we illustrate the example of $n$ distinct points on a line; here $\mathcal{Z}(\mathcal{A})$ is a real line with $n$ double points. More generally, $\mathcal{Z}(\mathcal{A})$ admits a natural map to $V$,

Received by the editors July 15, 2006 and, in revised form, September 14, 2006.

2000 Mathematics Subject Classification. Primary 52C35.

This author was partially supported by an NSF Postdoctoral Research Fellowship.

(C)2007 American Mathematical Society Reverts to public domain 28 years from publication 


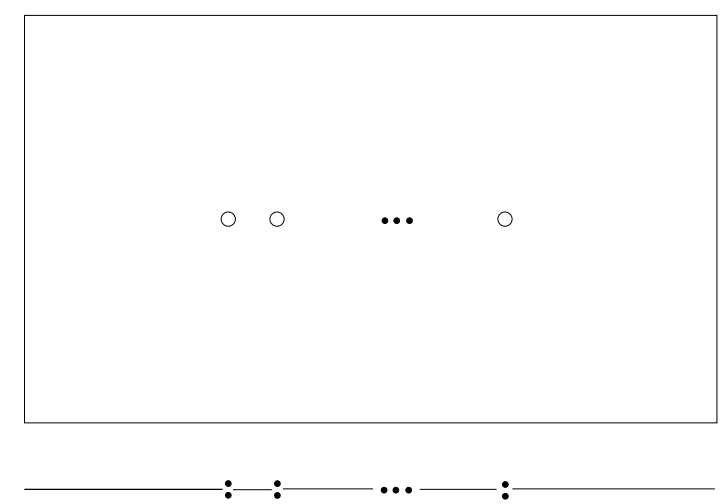

Figure 1 . $\mathcal{M}(\mathcal{A})$ and $\mathcal{Z}(\mathcal{A})$, where $\mathcal{A}$ consists of $n$ distinct points on a line.

and the number of points of $\mathcal{Z}(\mathcal{A})$ lying above a point $q \in V$ is equal to the number of chambers $C$ which contain $q$ in their closures.

In this particular example, it is clear that there is a map from $\mathcal{M}(\mathcal{A})$ to $\mathcal{Z}(\mathcal{A})$ given by compressing the imaginary axis, and that this map is a weak homotopy equivalence (see Remark 1.2). In the following theorem, which is our main result, we strengthen this observation, and generalize it to arbitrary arrangements.

Theorem 1.1. The complexified complement $\mathcal{M}(\mathcal{A})$ may be realized as a principal bundle over $\mathcal{Z}(\mathcal{A})$ in the category of real analytic prevarieties, with structure group naturally isomorphic to the additive group of the dual vector space $V^{\vee}$.

Remark 1.2. Since $\mathcal{M}(\mathcal{A})$ is isomorphic to the total space of a locally trivial fiber bundle over $\mathcal{Z}(\mathcal{A})$ with contractible fibers, the projection from $\mathcal{M}(\mathcal{A})$ to $\mathcal{Z}(\mathcal{A})$ is a weak homotopy equivalence, i.e., it induces isomorphisms on all homotopy and homology groups. This map is not an honest homotopy equivalence because it does not have a homotopy inverse; in particular, it admits no section.

Remark 1.3. Theorem 1.1 bears a strong similarity to the celebrated theorem of Salvetti $[\mathrm{Sa}$ which exhibits a simplicial complex $\operatorname{Sal}(\mathcal{A})$ that is homotopy equivalent to $\mathcal{M}(\mathcal{A})$. Salvetti's theorem offers some obvious advantages over Theorem 1.1; the space in question is a simplicial complex where one gets a homotopy equivalence rather than a weak homotopy equivalence, and the Salvetti complex is an invariant of the oriented matroid associated to $\mathcal{A}$. On the other hand, Theorem 1.1 has some advantages of its own. First, the space $\mathcal{Z}(\mathcal{A})$ is quite easy to visualize, perhaps more so than $\operatorname{Sal}(\mathcal{A})$. Second, we are able to work real analytically, rather than simply topologically. Finally, there is a canonical map from $\mathcal{M}(\mathcal{A})$ to $\mathcal{Z}(\mathcal{A})$, while the homotopy equivalence in Salvetti's theorem requires some arbitrary choices.

Remark 1.4. In the special case where the arrangement $\mathcal{A}$ is central and defined over the rational numbers, Theorem 1.1 may be interpreted in the world of hypertoric varieties, originally introduced by Bielawski and Dancer $[\mathrm{BD}$. (Bielawski and Dancer work exclusively with complex hypertoric varieties; for a treatment over arbitrary fields, see $[\mathrm{PW}$.) A real hypertoric variety is a real variety of dimension $2 d$ associated to a rational hyperplane arrangement of rank $d$, and it carries an action 
of the real algebraic torus $\left(\mathbb{R}^{\times}\right)^{d}$. Let $\mathcal{Y}(\mathcal{A})$ denote the open subset of this variety on which $\left(\mathbb{R}^{\times}\right)^{d}$ acts freely. One may show that $\mathcal{Y}(\mathcal{A}) /\{ \pm 1\}^{d}$ is isomorphic to $\mathcal{M}(\mathcal{A})$, and $\mathcal{Y}(\mathcal{A}) /\left(\mathbb{R}^{\times}\right)^{d}$ is isomorphic to $\mathcal{Z}(\mathcal{A})$. Hence $\mathcal{M}(\mathcal{A})$ is a principal bundle over $\mathcal{Z}(\mathcal{A})$ with structure group $\left(\mathbb{R}^{\times}\right)^{d} /\{ \pm 1\}^{d}$, which is analytically isomorphic to the additive group $\mathbb{R}$. Though we will not use the language of hypertoric varieties in our proof of Theorem 1.1, those familiar with hypertoric varieties will observe that the proof is closely guided by this interpretation.

Remark 1.5. Since $\mathcal{Z}(\mathcal{A})$ comes with an open cover $\left\{V_{C}\right\}$, it is natural to consider the Mayer-Vietoris spectral sequence associated to this cover. All multiple intersections of open sets are complements of real hyperplane arrangements, and therefore have cohomology only in degree zero. It follows that the cohomology groups of $\mathcal{Z}(\mathcal{A})$ are equal to the homology groups of the complex $E_{1}^{0, *}$, where $E_{1}^{0, q}$ is the direct sum of the degree zero cohomology groups of all $(q+1)$-fold intersections. Theorem 1.1 tells us that the cohomology groups of $\mathcal{Z}(\mathcal{A})$ are isomorphic to those of $\mathcal{M}(\mathcal{A})$, which are well understood by $\mathrm{OS}$. It would be interesting to see the complex $E_{1}^{0, *}$ arise in some independent combinatorial context.

Example 1.6. We conclude the first section with a detailed analysis of the simplest case, that of a single point on a line, which is the $n=1$ case in Figure 1. Here $\mathcal{M}(\mathcal{A})$ is isomorphic to $\mathbb{C}^{\times}$, and $\mathcal{Z}(\mathcal{A})$ is a real line with a double point at the origin. We will write down explicitly the action of the additive group $\mathbb{R}$ on $\mathcal{M}(\mathcal{A})$, see that the orbit space is isomorphic to $\mathcal{Z}(\mathcal{A})$, and check that the projection from $\mathcal{M}(\mathcal{A})$ to $\mathcal{Z}(\mathcal{A})$ is locally trivial. This will serve both as an illustration of Theorem 1.1 and as an important tool to apply toward the proof of the general case in Section 2.

First consider the action of $\mathbb{R}^{\times}$on $\mathbb{R}^{2} \backslash\{0\}$ given by the formula

$$
\lambda \cdot(x, y)=\left(\lambda x, \lambda^{-1} y\right) .
$$

The map from $\mathbb{R}^{2} \backslash\{0\}$ to $\mathbb{R}$ taking $(x, y)$ to $x y$ is surjective and $\mathbb{R}^{\times}$-invariant. The fiber of this map over a nonzero number consists of a single $\mathbb{R}^{\times}$orbit, while the fiber over zero consists of two orbits, namely the two coordinate axes. Hence the quotient of $\mathbb{R}^{2} \backslash\{0\}$ by $\mathbb{R}^{\times}$is a line with a double point at the origin, which we will call $\mathbb{D}$. The restriction of the action to the complement of either of the two coordinate axes in $\mathbb{R}^{2}$ induces a trivial $\mathbb{R}^{\times}$bundle over the quotient $\mathbb{R}$, therefore $\mathbb{R}^{2} \backslash\{0\}$ is a principal $\mathbb{R}^{\times}$bundle over $\mathbb{D}$, trivialized over the two copies of $\mathbb{R}$ in $\mathbb{D}$.

Now consider the quotient of this entire picture by the subgroup $\{ \pm 1\} \subseteq \mathbb{R}^{\times}$. We now obtain an action of

$$
\mathbb{R}^{\times} /\{ \pm 1\} \cong \mathbb{R}_{+} \cong \mathbb{R}
$$

on

$$
\left(\mathbb{R}^{2} \backslash\{0\}\right) /\{ \pm 1\} \cong \mathbb{C}^{\times} /\{ \pm 1\} \cong \mathbb{C}^{\times},
$$

where the isomorphism between $\mathbb{R}_{+}$and $\mathbb{R}$ is given by the logarithm, and the isomorphism between $\mathbb{C}^{\times} /\{ \pm 1\}$ and $\mathbb{C}^{\times}$is given by the map taking $\pm z$ to $i z^{2}$. (We include the factor of $i$ for technical reasons to simplify notation in the proof of the general case of Theorem 1.1.) Thus we obtain $\mathbb{C}^{\times}$as a principal $\mathbb{R}$ bundle over $\mathbb{D}$. In fact, the structure group of the bundle is naturally dual to the original line, hence we will denote the structure group $\mathbb{R}^{\vee}$.

For the sake of concreteness, let us write this action down in coordinates. To make clear the distinction between the action of $\mathbb{R}^{\vee}$ on $\mathbb{C}^{\times}$and the action of $\mathbb{R}^{\times}$ 
on $\mathbb{R}^{2} \backslash\{0\}$ given by equation (1), we will denote the $\mathbb{R}^{\vee}$ action with the symbol $*$. Given an element $z \in \mathbb{C}^{\times}$, choose a complex number $x+i y$ such that $z=i(x+i y)^{2}$. Then for $\xi \in \mathbb{R}^{\vee}$, we have

$$
\xi * z=i\left(e^{\xi} x+e^{-\xi} y i\right)^{2}=-2 x y+i\left(e^{2 \xi} x^{2}-e^{-2 \xi} y^{2}\right)=\operatorname{Re}(z)+i\left(e^{2 \xi} x^{2}-e^{-2 \xi} y^{2}\right) .
$$

Hence the action of $\mathbb{R}^{\vee}$ changes the imaginary part of $z$, and leaves the real part fixed. If the real part of $z$ is nonzero, then $x$ and $y$ are both nonzero, and the orbit $\mathbb{R}^{\vee} * z$ is equal to the vertical real line through $z$. On the other hand, if $z$ is purely imaginary, then either $x$ or $y$ is zero, and the orbit $\mathbb{R}^{\vee} * z$ is equal to the component of the punctured imaginary axis containing $z$.

Remark 1.7. It is easy to become confused by the changes of variables in Example 1.6. We will always adhere to the convention that when we use the letters $x, y$, and $z$, we have $z=i(x+i y)^{2}$. If we need letters to refer to the real and imaginary parts of $z$, as we will in Lemma 2.1, we will use the notation $z=a+i b$.

\section{ProOF}

We now turn to the proof of the general case of Theorem 1.1. Consider the affine linear map

$$
f: V \rightarrow \mathbb{R}^{n}
$$

given by the functions $f_{i}$ which define the hyperplanes of $\mathcal{A}$. The complexification $f^{\mathbb{C}}$ of $f$, given by the equation $f^{\mathbb{C}}(v+i w)=f(v)+i(f(w)-f(0))$, induces a closed embedding of $\mathcal{M}(\mathcal{A})$ into $\left(\mathbb{C}^{\times}\right)^{n}$. By Example 1.6, $\left(\mathbb{C}^{\times}\right)^{n}$ is a principal bundle over $\mathbb{D}^{n}$ with structure group $\left(\mathbb{R}^{n}\right)^{\vee}$. Let $\pi$ denote the projection from $\left(\mathbb{C}^{\times}\right)^{n}$ to $\mathbb{D}^{n}$.

Lemma 2.1. The image of $\mathcal{M}(\mathcal{A})$ under $\pi$ is isomorphic to $\mathcal{Z}(\mathcal{A})$.

Proof. Let us write $\mathbb{D}=\mathbb{R}^{\times} \cup\{p, m\}$, where $p$ and $m$ stand for plus and minus. Then the projection from $\mathbb{C}^{\times}$to $\mathbb{D}$ is given by sending $a+i b$ to $a$ if $a$ is nonzero, and otherwise to $p$ or $m$, depending on the sign of $b$.

Let $\tilde{a}=\left(\tilde{a}_{1}, \ldots, \tilde{a}_{n}\right)$ be a point of $\mathbb{D}^{n}$ lying over a point $a=\left(a_{1}, \ldots, a_{n}\right) \in \mathbb{R}^{n}$. Then $\tilde{a}$ lifts to an element $a+i b$ of $\mathcal{M}(\mathcal{A})$ if and only if $a$ lies in the image of $f$, and there exists $b=\left(b_{1}, \ldots, b_{n}\right) \in \mathbb{R}^{n}$ satisfying the following conditions:

$$
\begin{aligned}
& \text { (i) } \quad b+f(0) \text { is in the image of } f ; \\
& \text { (ii) if } \tilde{a}_{j}=p, \text { then } b_{j}>0 ; \\
& \text { (iii) if } \tilde{a}_{j}=m, \text { then } b_{j}<0 .
\end{aligned}
$$

Consider the map $\tilde{f}_{C}: V_{C} \rightarrow \mathbb{D}^{n}$ lifting the map $f: V \rightarrow \mathbb{R}^{n}$, defined by the property that if $f_{j}(q)=0$, then the $j^{\text {th }}$ coordinate of $\tilde{f}_{C}(q)$ is determined by the sign of $f_{j}$ on $C$. These maps glue together to define an inclusion

$$
\tilde{f}: \mathcal{Z}(\mathcal{A}) \rightarrow \mathbb{D}^{n} .
$$

Furthermore, $\tilde{a}$ is in the image of $f_{C}$ if and only if $a$ is in the image of $f$ and $C$ is contained in the set

$$
S(\tilde{a}):=\bigcap_{\tilde{a}_{j}=p} f_{j}^{-1}\left(\mathbb{R}_{+}\right) \cap \bigcap_{\tilde{a}_{j}=m} f_{j}^{-1}\left(\mathbb{R}_{-}\right) .
$$

It follows that $\tilde{a}$ is in the image of $\tilde{f}$ if and only if $a$ is in the image of $f$ and $S(\tilde{a})$ is nonempty. Suppose that $a=f\left(q_{0}\right)$ for some point $q_{0} \in V$. Then the map

$$
q \mapsto f(q)-f\left(q_{0}\right)=f\left(q-q_{0}\right)-f(0)
$$


takes $S(\tilde{a})$ bijectively onto the set of points $b \in \mathbb{R}^{n}$ satisfying conditions $(i),(i i)$, and (iii) above; hence $S(\tilde{a})$ is nonempty if and only if such a $b$ exists. This proves that the image of $\tilde{f}$ coincides with the image of $\pi$ restricted to $\mathcal{M}(\mathcal{A})$.

Let

$$
\mathcal{N}(\mathcal{A})=\pi^{-1}(\pi(\mathcal{M}(\mathcal{A})))=\left(\mathbb{R}^{n}\right)^{\vee} * \mathcal{M}(\mathcal{A}) \subseteq\left(\mathbb{C}^{\times}\right)^{n},
$$

where the second equality follows from the fact that the fibers of $\pi$ are precisely the orbits of $\left(\mathbb{R}^{n}\right)^{\vee}$.

Corollary 2.2. $\mathcal{N}(\mathcal{A})$ is a principal bundle over $\mathcal{Z}(\mathcal{A})$ with structure group $\left(\mathbb{R}^{n}\right)^{\vee}$.

Let $W \subseteq\left(\mathbb{R}^{n}\right)^{\vee}$ be the set of linear forms on $\mathbb{R}^{n}$ which are constant on the affine subspace $V$. (Equivalently, $W$ is the kernel of the dual of the linearization of $f$.) Consider an element $z \in\left(\mathbb{C}^{\times}\right)^{n}$, and choose $x, y \in \mathbb{R}^{n}$ such that $z_{j}=i\left(x_{j}+i y_{j}\right)^{2}$ for all $j \in\{1, \ldots, n\}$. We define a function $\rho_{z}: W \rightarrow \mathbb{R}$ by the formula

$$
\rho_{z}(\xi)=\|\xi * z\|_{\mathbb{C}^{n}}=\left\|e^{\xi} \cdot(x, y)\right\|_{\mathbb{R}^{2 n}}^{2},
$$

where $e^{\xi} \in \exp (W) \subseteq\left(\mathbb{R}^{\times}\right)^{n}$ acts coordinatewise as in equation (1), and the norms are the Euclidean norms on $\mathbb{C}^{n}$ and $\mathbb{R}^{2 n}$, respectively. Given a nonzero element $\xi \in W$, we compute the directional derivative of $\rho_{z}$ along $\xi$ :

$$
\frac{d}{d t}\|\exp (t \xi) \cdot(x, y)\|^{2}=\sum_{j=1}^{n} \frac{d}{d t}\left\|\left(e^{t \xi_{j}} x_{j}, e^{-t \xi_{j}} y_{j}\right)\right\|^{2}=\sum_{j=1}^{n} 2 \xi_{j}\left(e^{2 t \xi_{j}} x_{j}^{2}-e^{-2 t \xi_{j}} y_{j}^{2}\right) .
$$

Evaluating at $t=0$, we obtain

$$
\left.\frac{d}{d t} \rho_{z}(t \xi)\right|_{t=0}=2\langle\xi, \operatorname{Im}(z)\rangle,
$$

where $\langle\cdot, \cdot\rangle$ is the natural pairing between $\left(\mathbb{R}^{n}\right)^{\vee}$ and $\mathbb{R}^{n}$.

We now prove a pair of lemmas that are analogous to the main theorem of $\mathrm{KN}$, which lays the groundwork for the equivalence of quotients in symplectic and algebraic geometry. This idea is central to the perspective alluded to in Remark 1.4 .

Lemma 2.3. If $z \in \mathcal{N}(\mathcal{A})$, then $\xi$ is a critical point of $\rho_{z}$ if and only if $\xi * z \in \mathcal{M}(\mathcal{A})$.

Proof. Using the fact that $\rho_{z}(\xi)=\rho_{\xi * z}(0)$, we may immediately reduce to the case where $\xi=0$. An element $z$ of $\mathcal{N}(\mathcal{A})$ automatically has its real part contained in the image of $f$, hence it lies in $\mathcal{M}(\mathcal{A})$ if and only if $\operatorname{Im}(z)$ lies in the image of the linearization of $f$. This in turn is the case if and only if the imaginary part of $z$ pairs trivially with every $\xi \in W$ (this is how $W$ is defined). Equation (3) tells us that this happens if and only if zero is a critical point of $\rho_{z}$.

Lemma 2.4. If $z \in \mathcal{N}(\mathcal{A})$, then $\rho_{z}$ has a unique critical point.

Proof. By differentiating equation (2) we see that $\rho_{z}$ is convex, hence any critical point must be unique. What remains is to prove existence.

Let us consider the behavior of the right hand side of equation (2) as $t$ becomes very large. If $\xi_{j}>0$, then the $j^{\text {th }}$ term approaches positive infinity provided that $x_{j} \neq 0$, otherwise it remains bounded. Similarly, if $\xi_{j}<0$, then the $j^{\text {th }}$ term approaches positive infinity provided that $y_{j} \neq 0$, and is otherwise bounded. Hence 
the directional derivative of $\rho_{z}$ along $\xi$ at $t \xi * z$ is positive for large $t$ provided that there exists an index $j$ for which either

$$
\xi_{j}>0 \text { and } x_{j} \neq 0 \quad \text { or } \quad \xi_{j}<0 \text { and } y_{j} \neq 0 .
$$

If this condition is satisfied, then when $t$ is large, the gradient of $\rho_{z}$ restricted to the sphere of radius $t$ always points outward, hence $\rho_{z}$ must have a critical point somewhere on exp of the ball of radius $t$. Hence it will suffice to prove that there exists an index $j$ satisfying equation (4).

Recall that $z_{j}=i\left(x_{j}+i y_{j}\right)^{2}$. If $x_{j}=0$, then $z_{j}$ lies on the negative part of the imaginary axis; if $y_{j}=0$, then it lies on the positive part of the imaginary axis. Since $z \in \mathcal{N}(\mathcal{A})$, there exists $w \in \mathcal{M}(\mathcal{A})$ lying in the same $\left(\mathbb{R}^{n}\right)^{\vee}$ orbit as $z$. In particular, we have $\operatorname{Im}\left(w_{j}\right)<0$ whenever $x_{j}=0$, and $\operatorname{Im}\left(w_{j}\right)>0$ whenever $y_{j}=0$. Suppose that condition (4) fails for all $j$. Then $\xi_{j}$ and $w_{j}$ have opposite signs whenever $\xi_{j} \neq 0$, hence $\langle\xi, w\rangle \neq 0$. This contradicts the fact that $\xi \in W$ and $w \in \mathcal{M}(\mathcal{A})$.

Lemmas 2.3 and 2.4 combine to tell us that each $W$ orbit in $\mathcal{N}(\mathcal{A})$ contains a unique element of $\mathcal{M}(\mathcal{A})$. Thus

$$
\mathcal{M}(\mathcal{A}) \cong \mathcal{N}(\mathcal{A}) / W \cong \mathcal{N}(\mathcal{A}) \times_{\left(\mathbb{R}^{n}\right)^{\vee}} V^{\vee}
$$

which is the principal bundle over $\mathcal{Z}(\mathcal{A})$ induced from $\mathcal{N}(\mathcal{A})$ by the surjection

$$
\left(\mathbb{R}^{n}\right)^{\vee} \rightarrow\left(\mathbb{R}^{n}\right)^{\vee} / W \cong V^{\vee}
$$

This completes the proof of Theorem 1.1

\section{REFERENCES}

[BD] R. Bielawski and A. Dancer. The geometry and topology of toric hyperkähler manifolds. Comm. Anal. Geom. 8 (2000), 727-760. MR1792372 (2002c:53078)

[KN] G. Kempf and L. Ness. The length of vectors in representation spaces. Algebraic geometry (Proc. Summer Meeting, Univ. Copenhagen, Copenhagen, 1978), pp. 233-243, Lecture Notes in Math., 732, Springer, Berlin, 1979. MR555701 (81i:14032)

[OS] P. Orlik and L. Solomon. Combinatorics and topology of complements of hyperplanes. Invent. Math. 56 (1980), 167-189. MR.558866 (81e:32015)

[PW] N. Proudfoot and B. Webster. Arithmetic and topology of hypertoric varieties. To appear in J. Alg. Geom.

[Sa] M. Salvetti. Topology of the complement of real hyperplanes in $\mathbb{C}^{N}$. Invent. Math. 88 (1987), 603-618. MR884802 (88k:32038)

Department of Mathematics, Columbia University, New York, New York 10027 